\title{
LEIS: A reliable tool for surface composition analysis?
}

\author{
D. Primetzhofer ${ }^{a}$, S. N. Markin ${ }^{a}$, J. I. Juaristi ${ }^{b}$, E. Taglauer $^{\text {c }}$, and P. Bauer ${ }^{a}$ \\ ${ }^{a}$ Institut für Experimentalphysik, Johannes Kepler Universität Linz, A-4040 Linz, Austria. \\ ${ }^{b}$ Departamento de Fisica de Materiales, Facultad de Ciencias Quimicas, Apartado 1072, E-20080 \\ San Sebastian, Spain \\ ${ }^{c}$ Max-Planck-Institut für Plasmaphysik, EURATOM Association, D-85748 Garching bei München,
} Germany.

\begin{abstract}
Different single and polycrystalline surfaces of $\mathrm{Cu}$ and $\mathrm{Ag}$ have been investigated by Time-Of-Flight low-energy ion scattering using ${ }^{4} \mathrm{He}^{+}$ions. The fraction of ions that survived single scattering from the outermost surface layers, $\mathrm{P}^{+}$, was measured in different neutralization regimes. At low energies, a distinct difference in $\mathrm{P}^{+}$was observed for non-equivalent $\mathrm{Cu}$ crystal surfaces for projectiles backscattered in a single collision. The polycrystalline surface was found to exhibit similar neutralization behaviour as the (111) single crystal surface. At higher energies, $\mathrm{P}^{+}$shows a strong dependence on the angular orientation of the single crystal. The impact of these findings on quantitative surface composition analysis by LEIS is discussed.
\end{abstract}

\section{PACS numbers:}

\author{
34.50.Dy, 68.49.Sf, 79.20.Rf
}

\section{Keywords:}

Low energy ion scattering, single crystal, neutralization, $\mathrm{Cu}(100), \mathrm{Ag}(111)$, ion fraction

\section{Introduction}

In low-energy ion scattering (LEIS) usually noble gas ions are used to investigate the composition of the topmost atomic layers of a (solid) sample. The LEIS technique is thought to be extremely surface sensitive: this is partially because of the high scattering cross section but especially due to very effective neutralization which assures the signal to originate from the outermost atomic layers only [1]. Qualitative understanding of the underlying processes is available and necessary but not sufficient to obtain quantitative information on the surface composition. Therefore, a quantitative prediction of the neutralization behavior for different materials would be highly desirable to avoid time consuming calibration of measurements. These procedures employed usually rely on the experience that in LEIS neutralization efficiency is thought to be a property of the scattering centre and projectile only, without any or only minor influence of the chemical environment of the scattering atom. However, some evidence for an influence of the surface composition on the neutralization efficiency of the atomic species of interest was reported before $[2,3,4,5]$.

The commonly employed charge exchange model for neutralization of noble gas ions on metallic surfaces includes two distinct types of processes: Auger neutralization (AN) along the trajectory is possible at any primary energy [6]. The neutralization rate $-\mathrm{dP}^{+} / \mathrm{dt}$ depends on the Auger transition rate $\Gamma_{\mathrm{A}}$ via $-\mathrm{dP}^{+} / \mathrm{dt}=\mathrm{P}^{+} \cdot \Gamma_{\mathrm{A}}$. From this, the surviving probabilities $\mathrm{P}^{+}$in and $\mathrm{P}^{+}{ }_{\text {out }}$ for incoming and outgoing trajectories are obtained as

$\mathrm{P}^{+}{ }_{\mathrm{j}}=\exp \left[-{ }_{0} \int{ }^{\Delta \mathrm{tj}} \Gamma_{\mathrm{A}}(\mathrm{z}(\mathrm{t})) \mathrm{dt}\right]=\exp \left[-\left\langle\Gamma_{\mathrm{A}}\right\rangle \Delta \mathrm{t}_{\mathrm{j}}\right] \approx$

$\exp \left[-\left\langle\Gamma_{\mathrm{A}}\right\rangle \Delta \mathrm{z}_{\mathrm{j}} / \mathrm{v}_{\perp \mathrm{j}}\right] \equiv \exp \left[-\mathrm{v}_{\mathrm{cj}} / \mathrm{v}_{\perp \mathrm{j}}\right]$,

where $j$ stands for in or out, $\left\langle\Gamma_{A}\right\rangle$ denotes the transition rate averaged over the trajectory and $\Delta t$ is the time spent by the projectile in the region, where neutralization processes can occur, i.e. with a high density of conduction electrons. In Eq. (1) also the characteristic velocity $v_{c}$ is defined as a measure of 
neutralization efficiency. From Eq. (1) it is clear that AN scales with $\Delta \mathrm{t}$, which is approximately equivalent to scaling with the velocity component $v_{\perp}$ of the projectile normal to the surface. The fraction $\mathrm{P}_{\mathrm{AN}}{ }^{+}$of projectiles that have survived surface scattering without being neutralized by $\mathrm{AN}$ is given by $\mathrm{P}_{\mathrm{AN}}{ }^{+}=\mathrm{P}^{+}{ }_{\text {in }} \cdot \mathrm{P}^{+}{ }_{\text {out }}=$ $\exp \left[-\left\langle\Gamma_{A}\right\rangle\left(\Delta t_{\text {in }}+\Delta t_{\text {out }}\right)\right] \approx \exp \left(-v_{c} / v_{\perp}\right)$, with the abbreviation $1 / v_{\perp} \equiv 1 / v_{\perp \text { in }}+1 / v_{\perp \text { out }}$

In the "reionization regime", collision induced processes, i.e. collision induced neutralization $(\mathrm{CIN})$ and reionization $(\mathrm{CIR})$, become possible for a minimum distance between projectile and scattering centre smaller than a critical value $R_{\min }(E, \theta)$ due to the evolution of molecular orbitals [7], [8], [9]. In the collision between the projectile and a target atom, a minimum distance smaller than $R_{\text {min }}$ is reached if for a fixed scattering angle $\theta$ - the projectile energy $E$ exceeds a certain threshold $E_{\text {th }}$. The specific value of $E_{t h}$ depends on the atomic species of the collision partners and on the scattering angle $\theta$; e.g., for $\mathrm{He}^{+}$ scattered from $\mathrm{Cu}$ and $\theta=129^{\circ}, \mathrm{E}_{\mathrm{th}}=2100 \mathrm{eV}$ [1]. For $E>E_{\text {th }}, P^{+}$is therefore not a unique function of $v_{\perp}$, since for a given projectile target atom combination the probabilities for the collision induced processes, $P_{C I N}$ and $P_{C I R}$, depend on $E$ and $\theta$ instead of $v_{\perp}$. This implies that for given values of $v_{\perp}$ and $\theta$, there are two distinct $\mathrm{P}^{+}$values referring to different angles of incidence and exit, $\alpha$ and $\beta$, merging at an apex at $\alpha \approx \beta$ [10] with $\alpha$ and $\beta$ measured with respect to the surface normal.

Thus, for backscattering from surface atoms at $E>E_{t h}$, the ion fraction is obtained as the sum of two contributions, i.e. survivals and reionized projectiles [11]:

$$
\mathrm{P}^{+}=\mathrm{P}_{\text {in }}^{+}\left(1-\mathrm{P}_{\text {CIN }}\right) \mathrm{P}_{\text {out }}^{+}+\left(1-\mathrm{P}_{\text {in }}^{+}\right) \mathrm{P}_{\mathrm{CIR}} \mathrm{P}_{\text {out }}^{+}
$$

Note, that at typical conditions $\mathrm{P}_{\mathrm{CIN}}>\mathrm{P}_{\mathrm{CIR}}$ holds, so that $\mathrm{P}^{+}<\mathrm{P}^{+}{ }_{\text {in }} \cdot \mathrm{P}^{+}$out, where $\mathrm{P}^{+}{ }_{\text {in }} \cdot \mathrm{P}^{+}$out represents the probability of an ion not to be neutralized by $A N\left(P_{C I N}=P_{C I R}=0\right.$, in Eq. (2)).

In a very recent work [12 ] we presented strong differences in the deduced ion fraction $\mathrm{P}^{+}$for different single crystalline and polycrystalline surfaces of a pure element, i.e. $\mathrm{Cu}$ and explained these crystal effects by the available qualitative models described above. Furthermore, the influence of the crystal structure was taken into account. These findings are expected to have major impact on surface composition analysis using LEIS, and therefore we will discuss them in more details in this work. We further present additional data for $\mathrm{Ag}$ and show that it can be explained by the models developed in [12].

\section{Experiment}

The experiments were performed using the Time-Of-Flight- (TOF-) LEIS setup ACOLISSA [13] with a scattering angle $\theta$ of $129^{\circ}$ and a detector acceptance angle of $0.92^{\circ}$. The angular precision of the manipulator is $\pm 0.1^{\circ}$ and $\pm 0.2^{\circ}$ for polar and azimuth scans, respectively. The time resolution of the system is set to typically 10 to $25 \mathrm{~ns}$. A post acceleration voltage can be applied along part of the flight path between sample and detector to separate backscattered ions from neutrals. The primary beam current is set to 25 to 100 $\mathrm{nA}$ in full beam mode, yielding 5 to $20 \mathrm{pA}$ in the chopped beam mode. The beam current remains constant within $10 \%$ after thermal equilibration $(\sim 2 \mathrm{~h})$. At normal incidence, the beam spot on the sample was found to be smaller than $1 \mathrm{~mm}$ in diameter. From this the safe range of incident angles (measured with respect to the surface normal) follows ( $\alpha<$ $65^{\circ}$ ) ensuring that the whole irradiated spot is "visible" for the detector.

Polished crystals were purchased with a roughness below $0.03 \mu \mathrm{m}$ and a precision of the orientation of $\pm 0.1^{\circ}$. Polycrystalline films were produced by ex-situ evaporation. All surfaces were prepared by cycles of $3 \mathrm{keV}$ $\mathrm{Ar}^{+}$-sputtering. The single crystals were annealed at $\geq 400^{\circ} \mathrm{C}$. Auger Electron Spectroscopy did not show any surface impurities after cleaning and the crystal structure was checked by low-energy electron diffraction (LEED).

\section{Results and Discussions}

Single crystals offer the opportunity to limit backscattering in a single collision to the outermost atomic layer(s). In double alignment geometries, both, the incident beam and the detector are aligned with a low index crystal direction, therefore shadowing deeper layer atoms from the primary beam and blocking contributions from multiple scattering [14]. Consequently, a TOF-spectrum recorded for a single crystal under these conditions allows to determine the ion fraction $\mathrm{P}^{+}$from a direct comparison of the ion peak $A^{+}$and the surface peak of neutrals $A^{0}$, since they are formed by particles backscattered in a single collision from the surface layers [15], [16]. Different detection efficiencies for ions and neutrals must be taken into account (see Fig.1) [1].

For any other geometry the ion fraction $\mathrm{P}^{+}(\alpha)$ can be deduced by comparison of $\mathrm{A}^{+}\left(0^{\circ}\right)$ and $\mathrm{A}^{+}(\alpha)$ considering the change in available scattering centers which assures an equivalent dose of primary projectiles. A 
similar procedure [17] allows determination of $\mathrm{P}^{+}$for polycrystalline targets independent from the method proposed in [10]. This evaluation procedure is necessary since for both, single crystals in random orientation and polycrystalline targets, large background contributions from multiple scattering would make it very difficult to select single scattered neutrals from the TOF spectra (see Fig.1). The main advantage of this kind of analysis, possible in TOF-LEIS, is that uncertainties of the differential scattering cross section or inelastic losses do not contribute since they affect both scattered neutrals and ions in the same way.

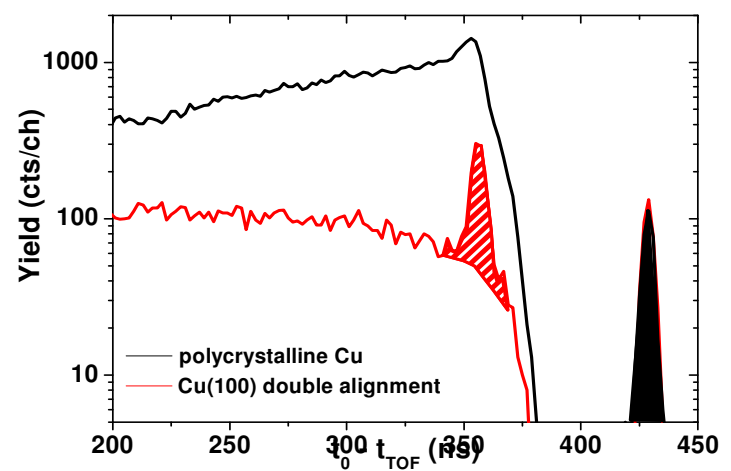

Fig.1: Experimental spectra are shown as a function of the inverse time-of-flight $t_{0}-t_{\text {TOF }}$, where $t_{0}$ is a constant and toF is the time-of-flight for $5 \mathrm{keV} \mathrm{He}{ }^{+}$ ions measured for normal incidence $\left(\alpha=0^{\circ}, \beta=51^{\circ}\right)$ on polycrystalline $\mathrm{Cu}$ (black line) and in double alignment conditions $\left(\alpha=0^{\circ}, \beta=51^{\circ}\right.$ azimuth in (001) direction) on a $\mathrm{Cu}(100)$ single crystalline surface (red line). The surface peak intensities of ions and neutrals after subtraction of a linear background are indicated as hatched and filled areas, respectively.

The system of $\mathrm{Cu}$ and $\mathrm{He}^{+}$is of special interest since the threshold energy of reionization is found to be rather high $(2100$ $\mathrm{eV}$ ), which allows to study $\mathrm{AN}$ in a wide energy range with our setup. We performed polar scans $\left(-15^{\circ}-70^{\circ}\right.$ with respect to the surface normal) for polycrystalline $\mathrm{Cu}$ and along the [001] and [112] azimuth direction for $\mathrm{Cu}(100)$ and $\mathrm{Cu}(110)$ respectively. The restriction to this azimuth directions is necessary, to obtain a signal originating from only one monolayer in double alignment geometry. The obtained ion fractions $\mathrm{P}^{+}$are presented in a semi-logarithmic plot in Fig. 2.

We found that the ion fraction for different surfaces are not equivalent but differ for energies below $1 \mathrm{keV}$ by at least a factor of 2. Furthermore, the ion fraction for polycrystalline $\mathrm{Cu}$ was found to be significantly lower than for the (100) and (110) surface. All the datasets presented in Fig. 2 exhibit a clear
$1 / v_{\perp}$ scaling thus proving that $A N$ is the dominating neutralization mechanism at $\mathrm{E} \leq 2$ $\mathrm{keV}$ in the system of $\mathrm{He}^{+}$and $\mathrm{Cu}$.

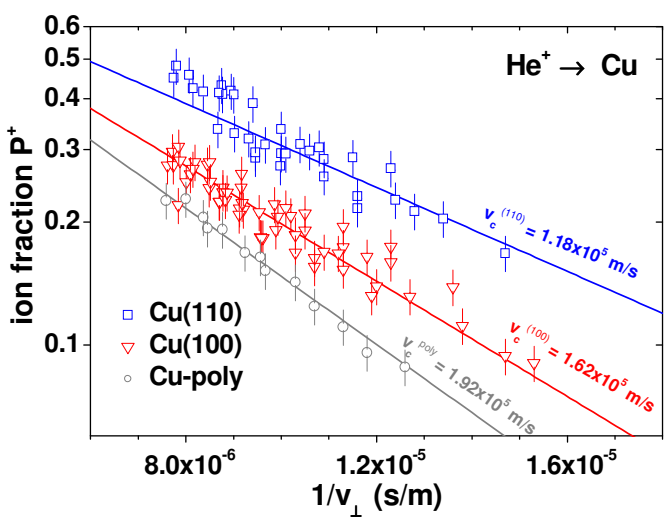

Fig.2: Ion fraction $\mathrm{P}^{+}$of $\mathrm{He}^{+}$scattered from $\mathrm{Cu}(110)$ (squares), $\mathrm{Cu}(100)$ (triangles) and polycrystalline $\mathrm{Cu}$ (circles) in the AN regime, as a function of $1 / \mathrm{v}_{\perp}$ $=1 / \mathrm{v}_{\perp \text { in }}+1 / \mathrm{v}_{\perp_{\text {out }}}$. Also shown are single exponential fits [see Eq. (1)] with characteristic velocity values as indicated in the figure.

It is a reasonable assumption to consider polycrystalline $\mathrm{Cu}$ to consist of (111) facets and therefore exhibiting a similar neutralization behavior as $\mathrm{Cu}(111)$. This is corroborated by measurements on $\mathrm{Ag}(111)$ which showed no statistically significant difference to polycrystalline Ag (Fig. 3).

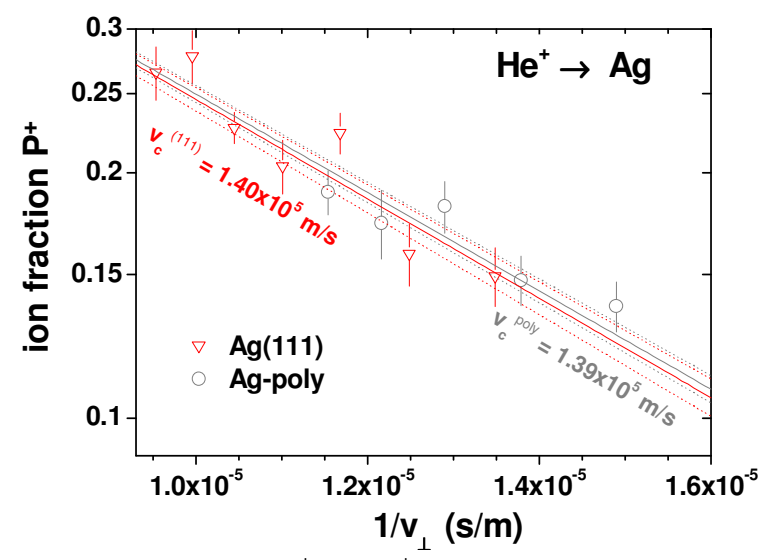

Fig.3: Ion fraction $\mathrm{P}^{+}$of $\mathrm{He}^{+}$scattered from $\mathrm{Ag}(111)$ (triangles), and polycrystalline $\mathrm{Ag}$ (circles) in the AN regime, as a function of $1 / v_{\perp}=1 / v_{\perp_{\text {in }}}+1 / \mathrm{v}_{\perp_{\text {out }}}$. Also shown are single exponential fits [see Eq. (1)] with characteristic velocity values as indicated in the figure.

In order to understand this crystal effect in the neutralization efficiency we theoretically limited the area where AN can take place to distances smaller than half an interlayer distance [18], [19] and assumed a homogeneous electron distribution. We calculated the time spent within this free electron gas using the MD simulation package KALYPSO [20 ]. This 
allowed us calculation of a mean Auger transition rate $\langle\Gamma\rangle$ from Eq.1. We found that $\langle\Gamma\rangle$ is virtually identical for all three $\mathrm{Cu}$ surfaces investigated, i.e. an AN rate of $2.2 \cdot 10^{15} \mathrm{~s}^{-1}$ is obtained within $1 \%$ accuracy. This implies that the neutralizing electrons in $\mathrm{Cu}$ behave like a free electron gas and are fully delocalized.

In Fig. 2 it can be noted that the observed crystal effect in the ion fraction for different crystal faces is diminishing with an increase in the primary energy. Therefore, we performed also measurements for various geometries at energies larger than the threshold energy of reionization $E_{\text {th }}$. The data acquisition and processing was done in a similar way as for the results shown for $\mathrm{E}<\mathrm{E}_{\mathrm{th}}$. Fig. 4a shows the apparent ion fraction observed for a $\mathrm{Ag}(111)$ single crystal in a polar scan performed at $6 \mathrm{keV}$. It is presented together with the linear fit of a dataset acquired for a polar scan at $E<E_{\text {th }}$ exhibiting clear $1 / \mathrm{v}_{\perp^{-}}$ scaling (see Fig.3). Similar to the data presented for $\mathrm{Cu}(100)$ in [12] the general behaviour of $\mathrm{P}^{+}$in the reionization regime is completely different from what is observed in the AN regime. Furthermore, the data is not in accordance with [10] measured on polycrystalline samples: although $\mathrm{P}^{+}$is again a double valued function of $1 / v_{\perp}$ no boomeranglike shape is found as observed for $\mathrm{P}^{+}$poly in the reionization regime. Consequently, Eq. (2) is not sufficient to describe the observations.

Similar to the high energy data presented for $\mathrm{Cu}(100)$ in [12] for the data shown in Fig. 4a the resulting $\mathrm{P}^{+}$values are virtually identical for double alignment conditions, i.e. for $\alpha \approx 20^{\circ}$ and $\alpha \approx 55^{\circ}$ (full symbols in Fig. 4a); outside double alignment conditions an increase in the ion yield by more than a factor of 3 is observed. For scattering from the outermost layers $\mathrm{P}^{+}$is almost independent of $\alpha$. This is because of the small efficiency of AN in comparison to collision induced processes.

The observed maximum in $\mathrm{P}^{+}$is caused by focussing collision cascades which direct a major part of the primary flux unto atoms in deeper layers leading to an increase of available scattering centres in subsurface layers and consequently of backscattered projectiles. This enhancement of backscattered intensity and its interplay with CIR and ineffective AN leads to the observed increase in $\mathrm{P}^{+}$due to reionization in a final large angle scattering process that may occur in or close to the surface [1]. This interpretation becomes more obvious when plotting the data as a function of $\alpha$ instead of $1 / v_{\perp}$ (see Fig. $4 b$ ).

In Fig. 4b, the minima at $\alpha=20^{\circ}$ and at $55^{\circ}$ can easily be recognized as channelling dips found exactly at the expected angular position for the investigated system [21]. The ion yield maximum at $\alpha=40^{\circ}$ corresponds to "random conditions", where focusing on deeper layer atoms is of importance. In fact, away from double alignment the neutral yield is even more strongly enhanced than the ion yield. This means that $\mathrm{P}^{+}$is actually significantly lower, if the increase in scattering centres from subsurface layers is considered, which is inherently linked to a loss in surface sensitivity. MARLOWE simulations revealed that - due to multiple scattering up to 10 crystal layers contribute to the measured scattering yield even at the final energy that corresponds to single scattering from atoms in the surface layer [15]. Consequently, the $\mathrm{P}^{+}$data presented in Fig. 4a represents only an apparent ion fraction since in any standard evaluation the unknown number of scattering centres for random single crystal orientation cannot be accounted for, but nevertheless a strong increase in the ion yield is observed.
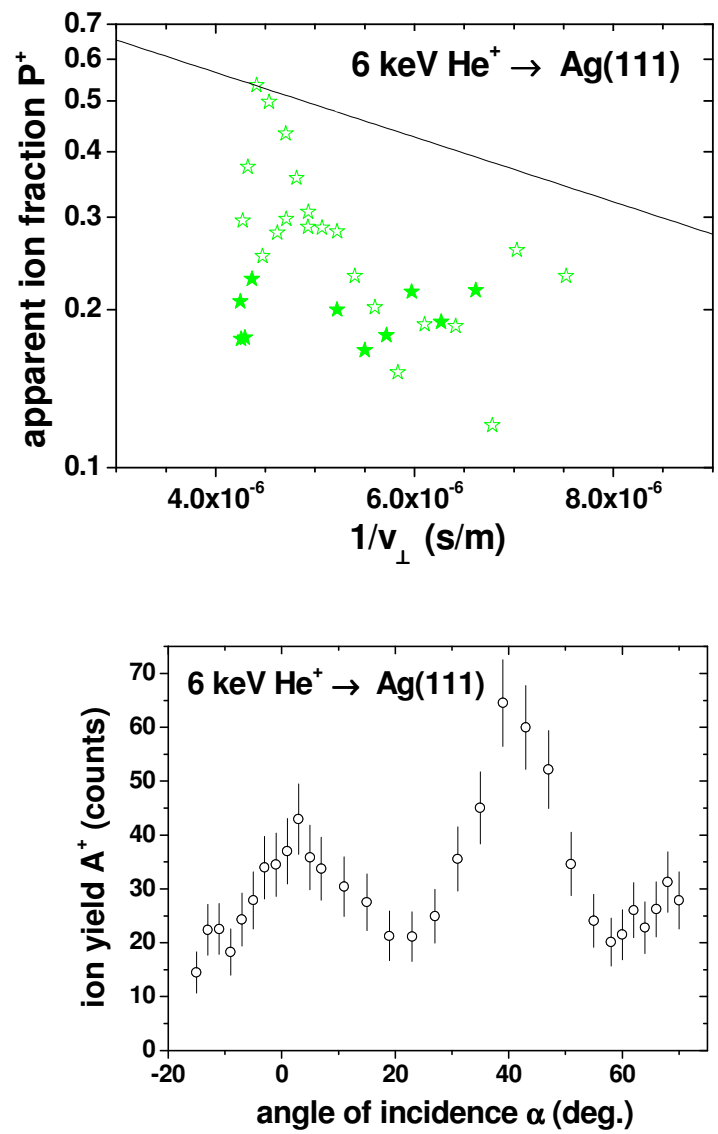

Fig.4: (a) Apparent ion fraction $\mathrm{P}^{+}$of $6 \mathrm{keV} \mathrm{He}^{+}$ scattered from $A g(111)$, as a function of $1 / v_{\perp}=$ $1 / \mathrm{v}_{\perp_{\text {in }}}+1 / \mathrm{v}_{\perp_{\text {out }}}$. Full asterisks refer to double alignment geometry, open asterisks to scattering out of double alignment. For comparison, the single exponential fit in the AN regime is shown (full line from Fig. 3). (b) Normalized ion yield $A^{+}$as a function of the angle of incidence $\alpha$ for the identical 
data set presented in Fig. 4(a), for $6 \mathrm{keV} \mathrm{He}{ }^{+}$ scattered from $\mathrm{Ag}(111)$.

We also performed measurements on polycrystalline $\mathrm{Cu}$ and $\mathrm{Ag}$ in the reionization regime. In contrast to the results presented in [10] we performed the evaluation of $\mathrm{P}^{+}$poly by comparison of the ratio of $A_{\text {poly }}^{+}(\alpha)$ and $\mathrm{A}_{\text {poly }}^{+}\left(0^{\circ}\right)$ to the ion yield of the single crystals in double alignment orientation. Supported by the previous findings we assumed the surface density of atoms of polycrystalline silver to be equivalent to the (111) single crystal surface. Similar to [10] we observed that in polar scans $\mathrm{P}^{+}$is a double valued function of boomeranglike shape. However, we found that the opening angle of the boomerangs is significantly smaller (not shown). The residual difference between the two distinct $\mathrm{P}^{+}$values corresponding to one value of $1 / \mathrm{v}_{\perp}$ can be explained by the remaining small contributions of $\mathrm{AN}$ where $\mathrm{P}^{+}{ }_{\text {out }}(\alpha)$ gives a higher survival probability for ions that leave the surface at angles close to $\alpha=0^{\circ}$.

The presented results have major impact on the reliability of surface composition analysis using LEIS since this technique is claimed to feature extreme surface sensitivity in combination with high precision measurements of atomic composition. The investigator has the choice whether to perform the experiment in the AN or the reionization regime. For both possibilities there are certain advantages and disadvantages. In the AN regime it can be assured that the information depth is limited to the outermost one or two atomic layers, independent of the used scattering geometry. However, one has to expect face dependent survival probabilities. Thus, calibration experiments using reference samples of pure elements will be unavoidable to predict the influence of these crystal effects. If experiments are performed in the reionization regime, the differences due to different surface orientations are vanishing. The main disadvantage is the loss of surface sensitivity, which can be partially restored only for single crystalline targets if a proper scattering geometry, i.e. double alignment conditions, is chosen. At high energies $E>>$ $E_{\text {th }}$, nevertheless background contributions to the ion peak will be observed and have to be considered in the evaluation. Anyway, if detection is done via $2 \pi$ integration - usually performed in experiments using an electrostatic analyzer - subsurface contributions to the ion yield never can be suppressed completely.

\section{Conclusions}

$\mathrm{He}^{+}$scattered from $\mathrm{Cu}$ is a simple system. Nevertheless, depending on the chosen crystal surface and the sample orientation differences up to a factor of 3 were observed in the apparent ion fraction. It is not clear yet whether the observed crystal effects are a peculiarity of the chosen system or a common property of transition metals. For surface composition analysis this means that large systematic errors might occur when the quantification is not performed properly. This rises the question, how "proper quantification" can be achieved. To our present knowledge, quantification of polycrystalline samples is less demanding since there structure effects can be excluded and the scattered intensity is azimuthally symmetric for perpendicular incidence of the ions. This is a most common case in current applications where electrostatic analyzer with $2 \pi$ azimuth acceptance is used for analysis. In this case, an analysis in the Auger neutralization regime appears attractive to make profit of its inherent high surface sensitivity. Before final conclusions can be drawn concerning the analysis of single crystalline samples, further investigations of various transition metals will be necessary.

\section{Acknowledgements}

This work was partly supported by the Austrian Science Fund (FWF) under contract number P16469-N08. Daniel Primetzhofer acknowledges a DOC-fellowship of the Austrian Academy of Science.

[1] H.H. Brongersma, M. Draxler, M. de Ridder and P. Bauer, Surface Science Reports 62 (2007) 63-109

[2] D.J. Godfrey, D.P. Woodruff, Surf. Sci. 105 (1981) 438.

[3] D.J. Godfrey, D.P. Woodruff, Surf. Sci. 105 (1981) 459.

[4] E. Taglauer, W. Englert, W. Heiland, and D. P. Jackson, Phys. Rev. Lett. 45(1980) 740.

[5] M. Beckschulte, E. Taglauer, Nucl. Instrum. Methods Phys. Res. Sect. B 78 (1993) 29.

[6] H.D. Hagstrum, Phys. Rev. 96 (1954) 336.

[7] R. Souda, M. Aono, C. Oshima, S. Otani, Y. Ishizawa, Surf. Sci. 150 (1985) L59.

[ 8 ] T.M. Thomas, H. Neumann, A.W. Czanderna, J.R. Pitts, Surf. Sci. 175 (1986) L737.

[9] N.P. Wang, E.A. Garcia, R. Monreal, F. Flores, E.C. Goldberg, H.H.Brongersma, P. Bauer, Phys. Rev. A 64 (2001) 012901.

[10] M. Draxler, R. Gruber, H.H. Brongersma, P. Bauer, Phys. Rev. Lett. 89 (2002) 263201. 
[11] L.K. Verhey, B. Poelsema and A.L. Boers, Nucl. Instr. Meth. 132 (1976) 565.

[12] D. Primetzhofer, S.N. Markin, E.Taglauer, P. Bauer, Phys. Rev. Lett. 100 (2008) 231201

[13] M. Draxler, S.N. Markin, S.N. Ermolov, K. Schmid, C. Hesch, R. Gruber, A. Poschacher, M. Bergsmann, P. Bauer, Vacuum 73 (2004) 39.

[14] Th. Fauster, Vacuum 38 (1988) 129

[15] D. Primetzhofer, S.N. Markin, R. Kolarova, M. Draxler, R. Beikler, E. Taglauer, P.Bauer, Nucl. Instr. Meth. B 258 (2007) 36

[16] D. Primetzhofer, S.N. Markin, M. Draxler, R. Beikler, E. Taglauer and P. Bauer, Surf. Sci. 602 (2008) 2921

[17] S.N. Markin, D. Primetzhofer, J.E. Valdés, E. Taglauer and P. Bauer, Nucl. Instr. Meth. B 258 (2007) 18

[18] P.J. Jennings, R.O. Jones and M. Weinert, Phys. Rev. B37 (1988) 6113.

[19] D. Valdés, E.C. Goldberg, J.M. Blanco and R.C. Monreal, Phys. Rev. B71 (2005) 245417.

[20] M.A. Karolewski, Nucl. Instr. Meth. B 230 (2005) 402

[21] Stereographic projections of fcc-surfaces, Daresbury Laboratories

http://www.dl.ac.uk/MEIS/stereographs/index.h $\underline{\mathrm{tm}}$ 